\title{
MIRAI Architecture for Heterogeneous Network
}

\section{Gang Wu and Mitsuhiko Mizuno, Communications Research Laboratory, Japan}

Paul J. M. Havinga, University of Twente, the Netherlands

\begin{abstract}
One of the keywords that describe next-generation wireless communications is "seamless." As part of the e-Japan Plan promoted by the Japanese Government, the Multimedia Integrated Network by Radio Access Innovation project has as its goal the development of new technologies to enable seamless integration of various wireless access systems for practical use by 2005 . This article describes a heterogeneous network architecture including a common tool, a common platform, and a common access. In particular, software-defined radio technologies are used to develop a multiservice user terminal to access different wireless networks. The common platform for various wireless networks is based on a wireless-supporting IPv6 network. A basic access network, separated from other wireless access networks, is used as a means for wireless system discovery, signaling, and paging. A proof-of-concept experimental demonstration system will be available in March 2002.
\end{abstract}

\section{INTRODUCTION}

Since the late 1980s, the use of second-generation mobile communication services has been explosively expanding worldwide. More and more wireless communication systems such as IEEE 802.11a/b wireless LAN, Bluetooth, IMT2000, and fixed wireless access (FWA) systems are coming into our lives, providing us more convenient ways to access the Internet and communicate with one another. Looking at the spectrum of frequencies ranging from several tens of megahertz to several tens of gigahertz, we find that there are dozens of (digital) communication systems. These ubiquitous systems are independently designed, implemented, and operated (Table 1) to meet different requirements for mobility, data rates, services, and so on. Some (if not all) of these systems can simultaneously pro- vide services at a specific geographic location, creating a heterogeneous wireless environment for users in overlaid service areas. The seamless integration of heterogeneous wireless systems will bring about a revolution in the wireless communications industry that will affect vendors, service/application/context providers, policy makers, and users.

With the adoption of IMT-2000 in Japan, researchers in both academia and industry have begun to show more and more interest in new-generation wireless communication networks. Japanese government adopted the socalled e-Japan Strategy in early 2001, including an explicit goal for wireless communications: to create an IPv6-based high-speed wireless Internet access environment and to enable seamless mobile communication services. The e-Japan Plan to achieve this goal requires developing fourth-generation mobile communication systems that will support a data rate as high as $100 \mathrm{Mb} / \mathrm{s}$ in a vehicular environment by 2010 and key technologies for the seamless integration of various wireless access systems for practical use by 2005. MIRAI (Japanese for future, and an acronym of Multimedia Integrated network by Radio Access Innovation), a project we at the Communications Research Laboratory are working on, is one of the Japanese national projects of the e-Japan Plan for the seamless integration of heterogeneous wireless systems.

Although the terms broadband and seamless are the main keywords for next-generation wireless networks, it is still unclear what these networks are. Figure 1 shows our understanding of the meaning of generation. We have no doubt about the meaning of second- and thirdgeneration systems/networks. However, fourthgeneration wireless networks should include not only new-generation cellular systems but also other new broadband wireless access systems such as ITS, HiSWAN, high-speed wire- 


\begin{tabular}{|c|c|c|c|}
\hline Mobility/frequency & Static & Pedestrian & Vehicle \\
\hline$<1 \mathrm{GHz}$ & & & $\begin{array}{l}2 \mathrm{G} \text { cellular } \\
\text { Two-way pager, MCA }\end{array}$ \\
\hline $1-3 \mathrm{GHz}$ & $\begin{array}{l}\text { WLAN }(802.11 \mathrm{~b}), \text { Bluetooth, } \\
\text { WPAN, VSAT }\end{array}$ & PHS, DECT & 2G/3G cellular, LEO \\
\hline $3-20 \mathrm{GHz}$ & WLAN (802.11a). BRAN, HiSWANa & BRAN, HiSWANa & 4G cellular, ITS \\
\hline Up to $60 \mathrm{GHz}$ & FWA, mm-wave WLAN, HAPS, HiSWANb & HAPS, HiSWANb & ITS, HAPS \\
\hline \multicolumn{4}{|c|}{$\begin{array}{l}\text { BRAN: Broadband radio access network; DECT: digital enhanced cordless telecommunications; HAPS: high } \\
\text { altitude platform station; HiSWAN: high-speed wireless access network; ITS: intelligent transportation sys- } \\
\text { tem; LEO: low earth orbit; MCA: multi channel access; mm-wave: millimeter wave; PHS: personal handy- } \\
\text { phone system; WLAN: wireless LAN; WPAN: wireless personal area network; VSAT: very small aperture } \\
\text { terminal. }\end{array}$} \\
\hline
\end{tabular}

Table 1. Various wireless access systems, especially in Japan.

less LAN, and high-altitude platform station (HAPS). That is, fourth-generation wireless networks should be heterogeneous networks that support multiple broadband wireless access technologies and global roaming across systems constructed by individual access technologies. Table 2 summarizes our understanding of a heterogeneous network.

Research on software-defined radio (SDR) technologies has shown that wireless physical layers can be created dynamically by introducing a code into programmable radio with tunable front-ends. An SDR-based user terminal may provide a common tool for users to access a heterogeneous network supported by different wireless access technologies. On the network side, a common platform is required to integrate different wireless access systems into a heterogeneous network. The Internet provides such a common platform, and the IPv6 technologies are potential technologies for network construction. Theoretically, with an SDR-based air-interfacereconfigurable user terminal and an IPv6-based

\begin{tabular}{lll}
$\begin{array}{l}\text { Network } \\
\text { with/in }\end{array}$ & $\begin{array}{l}\text { Same wireless } \\
\text { access technology }\end{array}$ & $\begin{array}{l}\text { Different wireless } \\
\text { access technologies }\end{array}$ \\
\hline $\begin{array}{l}\text { Same administrative } \\
\text { domain (company) }\end{array}$ & Homogeneous & $\begin{array}{l}\text { Heterogeneous } \\
\text { (limited services, } \\
\text { easy implementation) }\end{array}$ \\
\hline $\begin{array}{l}\text { Different administrative } \\
\text { domains (companies) }\end{array}$ & $\begin{array}{l}\text { Heterogeneous } \\
\text { (limited services, } \\
\text { complicated } \\
\text { implementation.) }\end{array}$ & $\begin{array}{l}\text { Heterogeneous } \\
\text { (general services, } \\
\text { most complicated } \\
\text { implementation) }\end{array}$ \\
\hline
\end{tabular}

Table 2. Definition of heterogeneous network.

heterogeneous network, a service can be delivered via the wireless access network most efficient for that service.

As shown in Table 1, different wireless access systems are distributed in spectrum. It takes time and consumes battery power to find an available and preferable wireless access system(s) at the place where the user is even with a reconfig-

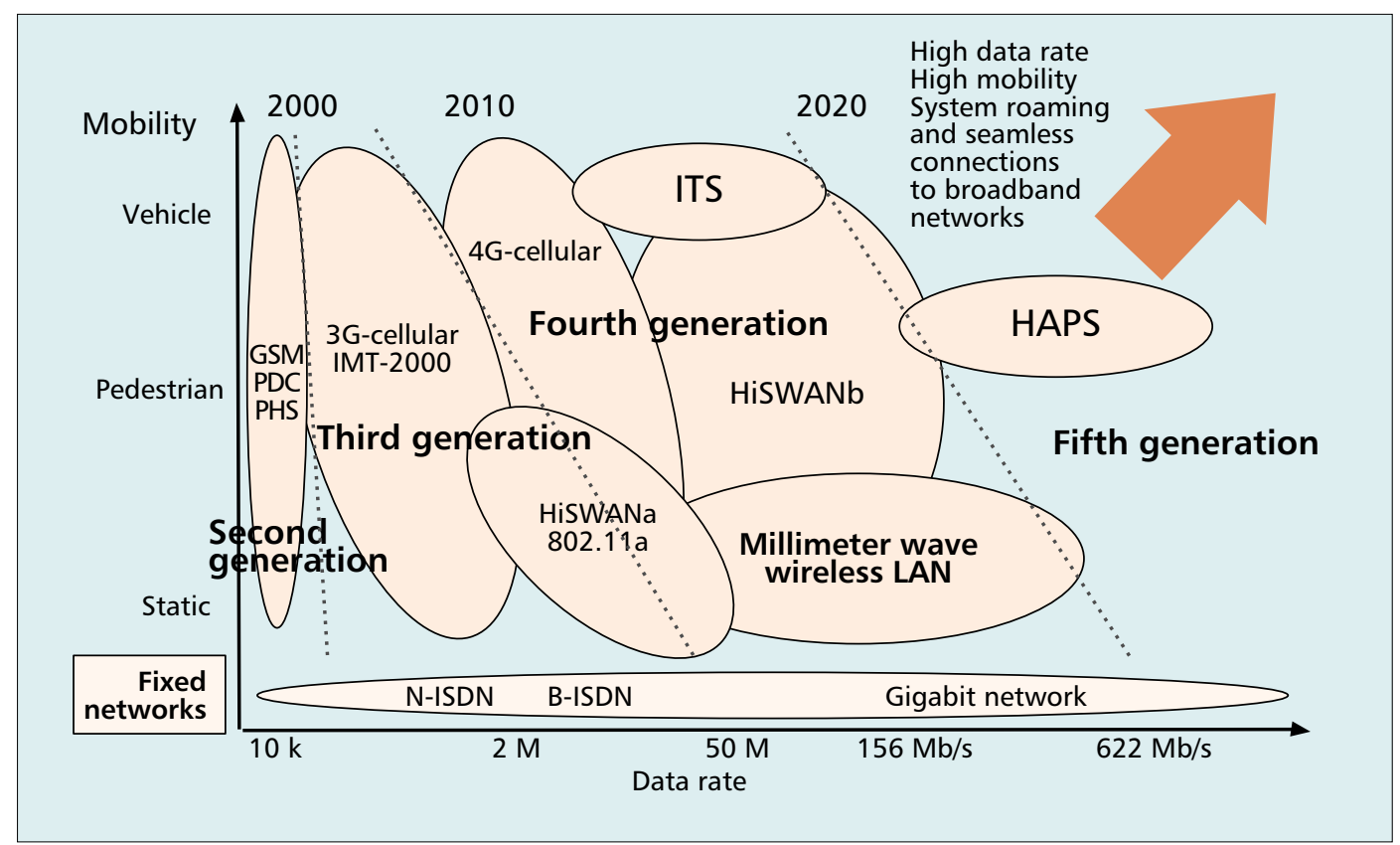

Figure 1. A definition of generations for wireless networks. 


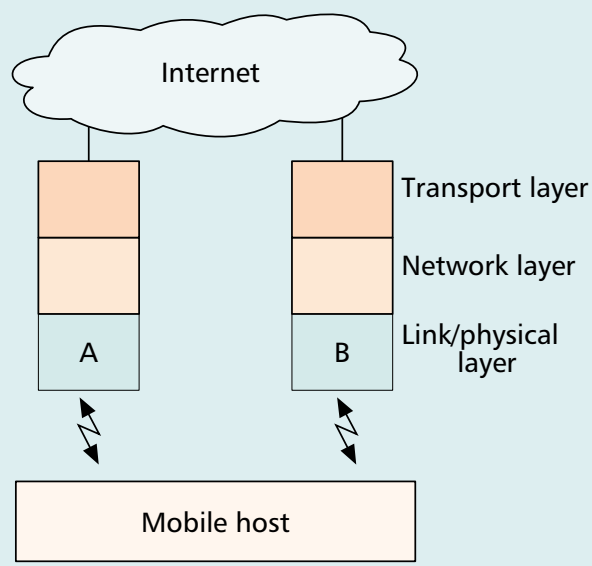

(a) Tunneled network

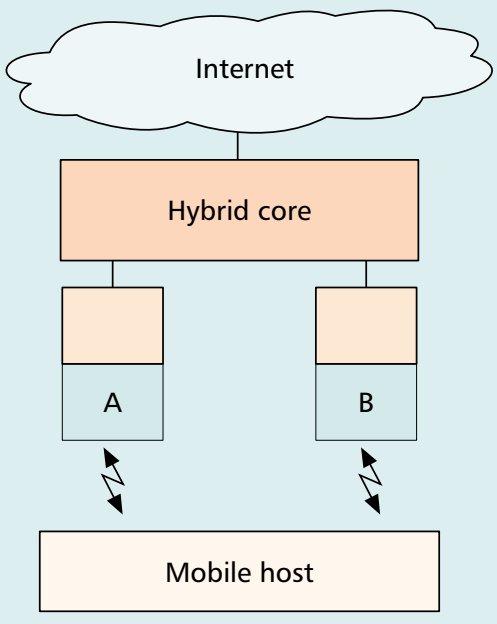

(b) Hybrid network

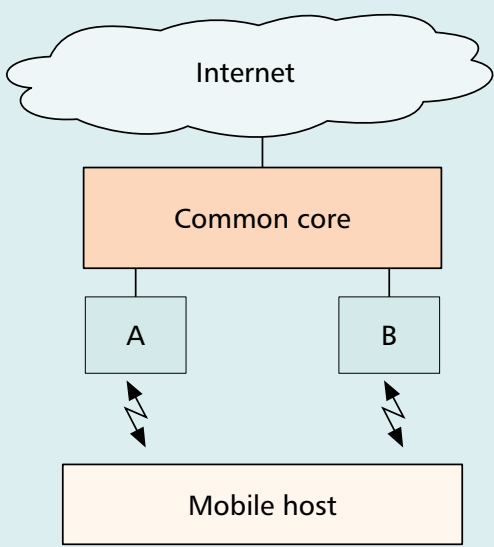

(c) Heterogeneous network

Figure 2. Architecture models.

urable SDR terminal. Supporting heterogeneous paging, vertical handoff, and so on is also technically quite difficult. We propose a basic access network that provides a common access function for all wireless access systems used for service delivery in a heterogeneous network to support heterogeneous paging, location update, wireless system discovery, vertical handoff, and so on. The basic access network may have a cellular configuration with a low-data-rate but reliable communication channel. Each base station will have a much wider service area than do the base stations in other wireless access systems.

With the goal to design a flexible and open architecture suitable for a variety of different wireless access technologies, as well as for applications with different QoS requirements and different protocols, the MIRAI project focuses on the research and development of a common tool, a common platform, and a common access. The solution is based on an SDR-based multiservice user terminal (MUT), an IPv6-based wireless supporting common core network $(\mathrm{CCN})$, and a basic access network (BAN). This article describes the MIRAI architecture.

The article is organized as follows. First, we introduce the concept of a heterogeneous network. Then we present an overview of related work in the field of micromobility and quality of service (QoS) mechanisms over the Internet. We present the main concepts of our architecture, describe the MIRAI architecture in detail, and finally describe the challenges we meet when implementing the architecture.

\section{MODELS OF Hetergeneous Networks}

There are several architectures using multiple radio access networks (RANs). The main models are illustrated in Fig. 2 by using two RANs, networks $\mathrm{A}$ and $\mathrm{B}$. The main distinction between these models is in the layer on which the RANs communicate. Many derivatives of these models are possible (e.g., $[1,2])$.

\section{TUNNELEd Networks}

In this model a user has a service agreement with the operators of several RANs independently. Based on a certain policy, the optimal network for the requested service is selected. The connectivity between networks is based on relatively high network layers of the Internet (i.e., transport or session layers), which increases the service latency. This system requires no modification to existing access networks. Moreover, all the networks have their own infrastructure (e.g., signaling, handover, and billing). This makes it very difficult for existing network systems to cooperate efficiently.

\section{HYBRID NETWORKS}

In this model we have a hybrid core that interfaces directly between RANs and the Internet. In this model RANs implement the network layer and the layers below. The advantages of this model include fewer duplicate functions and more advanced services at the network or data link layer (e.g., it can provide a better handover between RANs).

\section{Heterogeneous Networks}

In this model there is a $\mathrm{CCN}$ that deals with all network functionality and operates as a single network. Different RANs handle only those tasks that are specifically related to a certain radio access technology. In general, wireless access radio incorporates the physical and data link layers only. Communication between RANs belonging to the same $\mathrm{CCN}$ is based on lower network layers (link or network). This reduces the overhead and improves network performance. A major challenge here is that different RANs must converge, which requires much effort to standardize the networks and a commitment from business to support this standardization.

Note our differentiation between hybrid and heterogeneous networks. Various kinds of architectures are often referred to as hybrid [2]. We prefer to call them heterogeneous to stress 
the fact that there can be multiple networks simultaneously, all working together. Hybrid networks describe a more traditional view of having multiple networks from which one can be chosen for use.

\section{RELATED WORK}

Future wireless network infrastructures must support a wide variety of users, applications, and access needs. High-speed access can be achieved by using small cells. However, as the base station density increases, so will the handoff rates. Currently, related work is focusing on the routing and handoff aspects of wireless networks. The Mobile IP protocol [3] supports mobility transparently above the IP level and allows nodes to change their location. Mobile IP is generally seen as a macromobility solution; it is less well suited for micromobility management, in which a mobile host moves within a subnetwork. A typical example of micromobility is a handoff among neighbor wireless transceivers, each of which covers only a very small geographical area. Quite a few schemes have been proposed to support micromobility (e.g., Cellular IP [4], HAWAII [5]). The difference among all these schemes is related to the mechanisms used to route packets within a local (home or foreign) domain.

Related work on QoS over the Internet is mainly based on integrated services [6] and differentiated services [7]. Recently, there have been a number of initiatives specifically related to heterogeneous networks, but this research has just begun [1]. Other related research mainly focuses on hybrid network architectures or support for macromobility [8,9]. Given that asynchronous transfer mode (ATM) can support QoS, there has been much interest in developing wireless ATM technologies (e.g., [10]).

Current work merely provides solutions to roaming mobile hosts by supporting protocols for mobility. Heterogeneous networks may be used, but more in a traditional sense of selecting one or another. This is different from our view of heterogeneous networks, in which mobile hosts can communicate over one or more RANs simultaneously.

\section{CONCEPTS}

A major challenge for the future-generation wireless Internet is that the architecture will have to be very flexible and open, capable of supporting various types of networks, terminals, and applications.

\section{SYSTEM REQUIREMENTS}

The fundamental goal is to make the heterogeneous network transparent to users. Another goal is to design a system architecture that is independent of the wireless access technology. These considerations lead to the following requirements:

Multiservice user terminal (MUT) for accessing different RANs: Having multiple wireless airinterface modules (may be implemented by either multimode air interfaces or an SDR-based reconfigurable air interface), an MUT is capable of using one or more specific air interface(s) to access one or more available RAN(s).

Wireless system discovery: For an MUT at a specific geographic location to use the RAN that meets the user's physical capability as well as the utilization policy, it must discover how many RANs are available in that area. This is an important but sometimes difficult and time-consuming process. Generally, three discovery methods are used: distributed (MUT searches for available RANs), centralized (the network announces how many RANs are available), and a combination of both.

Wireless system selection: An important feature of a heterogeneous network is that an MUT can select, from a number of available RANs, the most appropriate one(s). The wireless system selection is based on both the user's utilization policy (e.g., price, data transmission rate, battery life, service grade) and the current traffic status of RANs (e.g., available bandwidth, congestion status). The result is that each service is delivered via the network most efficient for this service.

Mobility management: The system must enable QoS guaranteed seamless handover within the same RAN (horizontal) and among different RANs (vertical) through the development of corresponding technologies.

Location update and paging: The system must be RAN-independent and user-transparent; it must be secure and must enable low signaling load, integrated controlling and managing, and roaming-supported location update. Location update technologies should enable heterogeneous paging for MUTs.

Simple, efficient, scalable, low-cost: All these requirements are closely related to each other. These requirements are of particular importance in future picocellular networks in which one access point should support up to several hundred megabits per second. It is not efficient to have many complex access points.

Energy-efficient: We expect that wireless IP communicators will be switched on, ready for service, and constantly reachable by the Internet. This implies that such functions as maintaining location information and wireless system discovery should be energy-efficient (and bandwidthefficient). Cellular systems employ the notion of passive connectivity to reduce the power consumption of idle mobile hosts.

Secure: Mobile systems face a number of security problems that do not exist in their stationary counterparts. Mobile hosts must update their location while moving. These location messages make impersonation possible unless the systems are secure. In systems and applications where seamless handover is of primary importance, session keys used by mobile hosts must be promptly available at the new base station (in the same or a different RAN) during handoff.

QoS support: End-to-end QoS mechanisms should be available. Since RANs provide services specialized for some service, QoS in heterogeneous networks is of primary importance. End-to-end QoS implies that there must be interoperation with local QoS mechanisms, and that lower layer protocols (link and physical layers) should be "aware" of the traffic characteristics to meet different requirements for QoS.
Research on

SDR technologies

has shown that

wireless physical

layers can be

created

dynamically by

introducing a

code into

programmable

radio with

tunable

front-ends. 


In developing our
architecture, we
try to the extent
possible to build
upon the existing
protocols to
minimize the
required effort,
and to
ensure system
compatibility with
existing protocols
and applications.

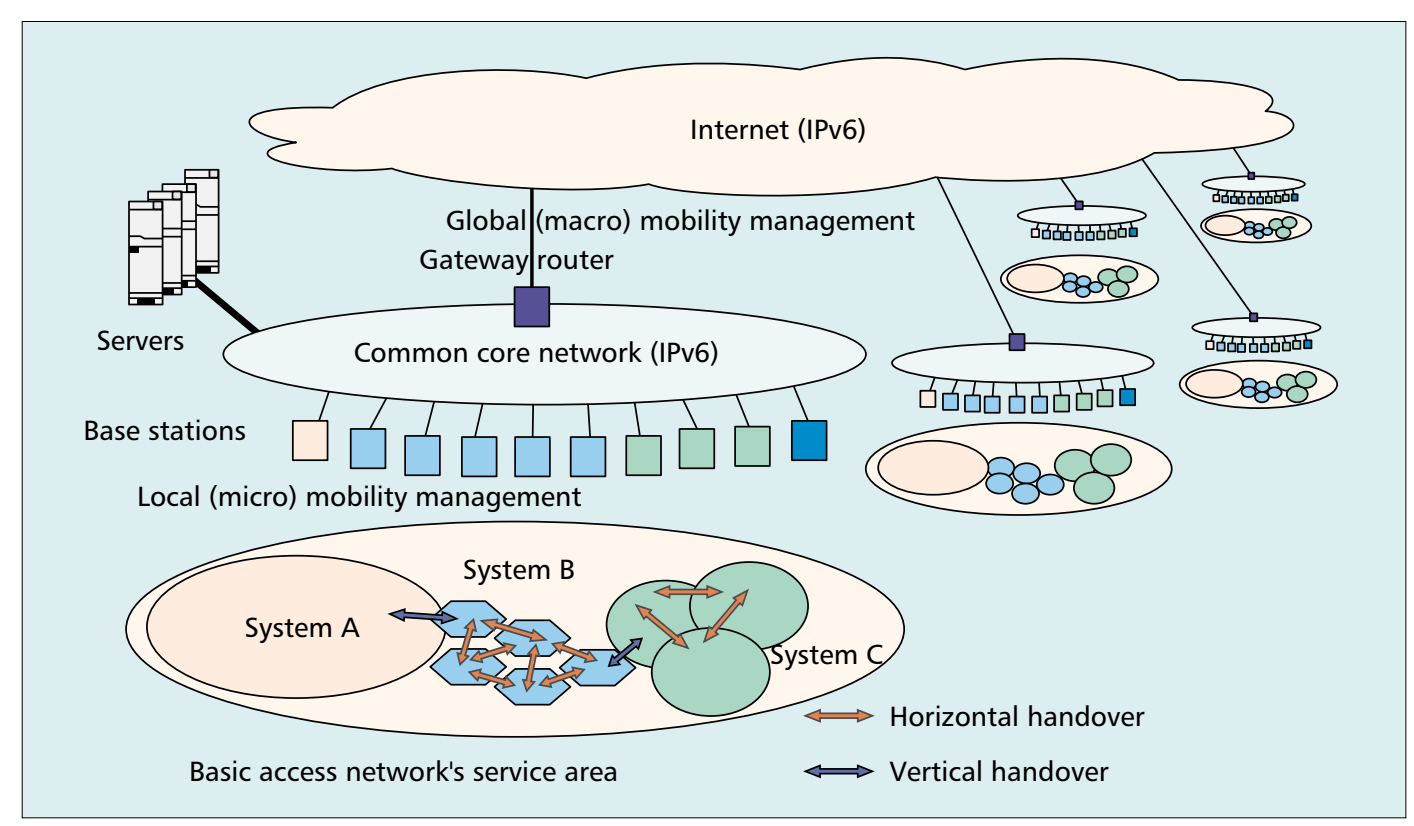

Figure 3. The MIRAI network concept.

Personal mobility: Personal mobility in heterogeneous networks is more important than in homogeneous ones. A user with a personal ID should be able to access different RANs.

It should be noted that some of these requirements are closely related to each other. Finding a solution for one requirement may provide solutions for others. In developing our architecture, we try to the extent possible to build on the existing protocols to minimize the required effort, and to ensure system compatibility with existing protocols and applications.

\section{BASIC ENTITIES}

Our solution is based on three major entities:

ICommon core network (CCN). This can be a managed IPv6 network providing a common platform through which all MUTs will communicate with correspondent nodes in the Internet. In principle all access points of RANs are connected to this network. The network provides QoS-guaranteed routing and seamless handover among RANs. This enables natural integration of various heterogeneous networks. The main functional entity of the $\mathrm{CCN}$ is a resource manager, which coordinates traffic distribution and selects the most appropriate RAN. It has a common database for managing users' profiles through entries such as authentication, location, preferred access system, billing, policy, and users' terminal capabilities.

IBasic access network (BAN). It provides a common control/signaling channel to enable all MUTs to access the common platform. The network is basically used to provide location update and paging and support wireless system discovery and vertical handoff for all other wireless systems. Consisting of base stations and basic access components (BACs, terminals), the BAN will have a broad coverage area, preferably larger than that of the RANs it supports, and a reliable communication means for signaling transmission, where a high data rate is not necessary.

IMultiservice user terminal (MUT). The MUT is equipped with a multi-radio system. All terminals have a BAC to communicate with the BAN. Apart from this radio system, an MUT is equipped with one or more radio subsystems to access the CCN. These subsystems are essentially (or preferably) based on SDR technologies, which allow an MUT to adapt its radio hardware to the wireless infrastructure available and required.

\section{THE NetWork Model}

The MIRAI architecture provides communication between mobile hosts and correspondent nodes in the Internet. Figure 3 illustrates the network configuration. The main component is the base station, which serves as a wireless access point and interfaces with the $\mathrm{CCN}$. CCNs are connected to the Internet via gateway routers. A $\mathrm{CCN}$ provides services for several RANs. In general, the RANs will overlap, and a mobile host can have access to several RANs in one location. The area covered by these wireless networks can be quite large.

Mobile IPv6 is the envisioned protocol for connecting CCNs and providing global (macro) mobility management. In a CCN-managed area, fast handover between base stations often belonging to different RANs with high-speed wireless access requires local (micro) mobility management. Mobile hosts attached to a base station use the IP address of the gateway as their Mobile IP care-of address. Inside a CCN, mobile hosts are identified by their home address. Base stations are connected to (or integrated with) a regular IP forwarding engine. These engines are connected through some network topology that allows packets to be transmitted between the base stations and the gateway.

Note that, although in our concept the base station is equal to a wireless access point, this is not a strict requirement. Wireless access providers may want to use their own network of interconnected access points, and share one base station to connect to the core network. An 
important concept in our architecture is simplicity, which enables low-cost implementation of the network. The concept of a $\mathrm{CCN}$ and a separate BAN offers providers of wireless services the possibility to set up an infrastructure without a huge investment of resources. New providers can easily connect to the core network, provided they use a correct interface. They do not need to have their own infrastructure ready before they can start their business, but instead can use the infrastructure provided by the core and the BAN. All they have to do is develop their wireless service and concentrate on the wireless access only. The infrastructure generally needed to set up a whole new service is already provided by the architecture. This includes both technical issues (e.g., setting up an interconnection network between base stations enabling routing and handoff, and providing Internet access) and business issues (e.g., billing and managing consumer profiles). The components they have to build are base stations and an access mechanism for terminals. In general, the access mechanism can be a software module suitable for SDR.

A consumer can have a contract with a $\mathrm{CCN}$ provider and buy various services (provided by a RAN) from the provider. If the consumer has a contract that enables him to use multiple services, the system and the user can select the most appropriate service. Access networks can also be combined to increase the available capacity. Different access networks can be used for the uplink and downlink traffic. This can be advantageous for many user applications such as Web browsing and e-mail, which in many cases are asymmetrical resulting in more downlink than uplink bandwidth. The result is that each service is delivered via the network that is most efficient (in many ways) to support the service. In effect, the consumer is unaware of the wireless technologies used to provide the service.

\section{Miral ARChitecture}

In this section we will introduce the functional entities of our architecture and the protocols used.

\section{OVERVIEW}

The architecture as depicted in Fig. 4 is composed of four major building blocks: a mobile host, RANs, a CCN, and an external network (or the Internet). Within the external network, there are correspondent nodes (CNs). One or more gateway routers (GRs) connect the external network to the CCN. The external network uses Mobile IPv6 is assumed. The GR detunnels tunneled packets destined to a mobile host and forwards them to a base station. Two important functional entities within the CCN are a resource manager (RM) and a mobility manager (MM). They are primarily responsible for traffic distribution and mobility-related problems.

The CCN supports communication with the base stations, and thus with RANs. A base station interface (BSI) is primarily used to provide a uniform access mechanism for the base stations to access the CCN. The BSI can be a component of a base station. The base stations (BSs) deal

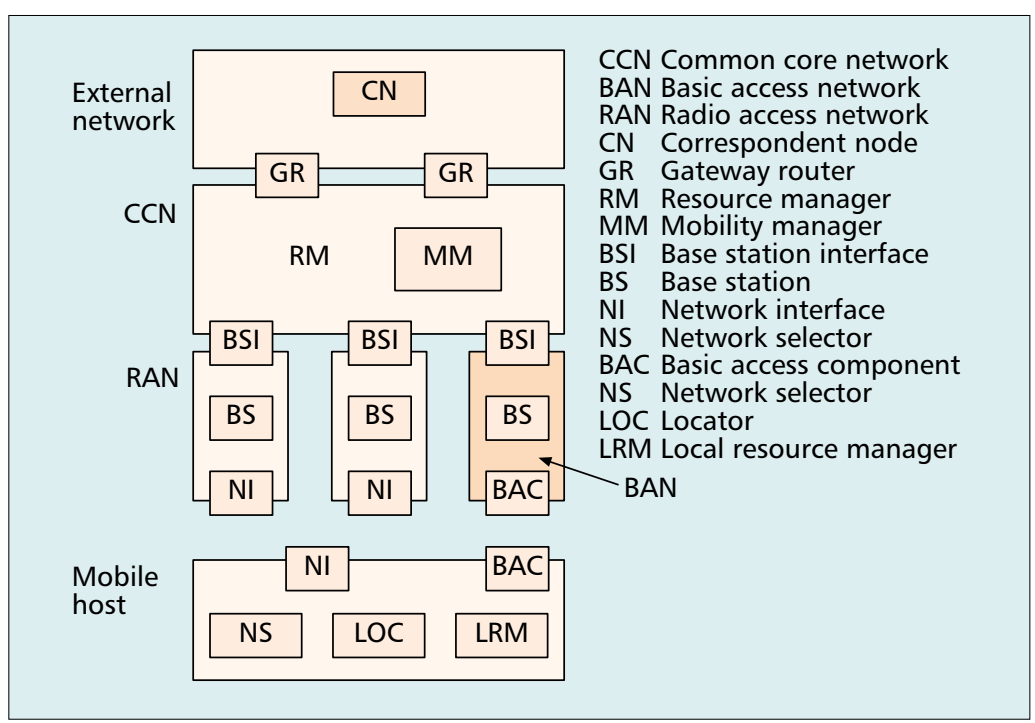

Figure 4. Hetereogeneous system architecture.

with wireless access problems in the normal link layer and collect status information of the wireless network they support. They use a network interface (NI) to access the network.

All mobile hosts have a BAC to communicate with the BAN. Besides this interface, mobile hosts also have an NI. However, in contrast to the NI of the BSs, this interface is generally based on SDR technologies to enable it to use multiple RANs. A network selector (NS) communicates with the RM to tune the radio for the RANs to use. A network selection control protocol is used to enable the proper selection of an access network. A locator (LOC) provides the $\mathrm{RM}$ with information on the location of mobile hosts. A local resource manager (LRM) deals with the local resources of the terminal and interacts with the RM at the CCN.

\section{FUNCTIONAL ENTITIES OF THE COMMON CORE NETWORK}

The main goal of our architecture is to integrate different access technologies into a common architecture. Through this integration, the system can be used efficiently and a mobile user can receive the services it requested. To achieve this goal, the main tasks to be fulfilled by the architecture are resource management to coordinate traffic distribution in the system and mobility management to support roaming mobile hosts.

The RM is thus responsible for resource allocation and admission control to support traffic distribution in the CCN. It selects a RAN that can provide the service requested by the mobile host in the most efficient way. In essence, it combines multiple wireless access systems and exploits their specific strength to provide services in a spectrum-efficient way [11]. Another task of the RM is to interact with IP QoS architectures (e.g., Intserv and Diffserv) that may be used in the external network. This is merely a mapping between QoS parameters of both worlds. We envision using several basic classes in the core network (e.g., best effort, real-time, adaptive). This mechanism enables a wireless link to prop- 


The main goal of
our architecture
is to integrate
different access
technologies into
a common
architecture.
Through this
integration, the
system can be
used efficiently
and a mobile user
can receive the
services it
requested.

erly support IP packets with varying IP QoS parameters. This functional entity is located in the network layer.

The RM enables service selection by using certain criteria. These criteria originate from various sources: the mobile host (i.e., the LRM), mobile user, applications, and BSs. Specific inputs are:

- QoS requirements of sessions

- User preferences such as cost and a preferred RAN

- Terminal capabilities such as supported access networks, protocols, and available resources

- Status (i.e., available resources) of the $\mathrm{CCN}$ and RANs

- Location of the mobile host

The RM should also incorporate costs involved in changing access networks (e.g., costs involved in reconfiguration of the software radio). This management task is by no means trivial, especially with mobiles roaming quickly through the region.

The mobility manager (MM) deals with all mobility-related issues. It keeps track of the location of mobiles, and determines which access networks are available to a mobile host at a certain location. The RM uses this information. The other main task of the MM is to provide handoffs, both local within the $\mathrm{CCN}$ and for the external network (based on Mobile IPv6). To provide these handoffs, it needs to interact with the RM. The MM is located at the network layer and operates in the CCN. If a mobile host moves within the core network, the mobility is transparent to the network layer, and the system tries to maintain IP flows and IP QoS parameters. In the case of inter-core-network mobility, reservations are recreated, due to which packets may be transmitted as best effort traffic.

\section{FUNCTIONAL ENTITIES OF THE BASIC ACCESS NETWORK}

In our architecture we use two separate networks: the BAN for common signaling-related traffic, and the $\mathrm{CCN}$ for data and signaling traffic related to individual RANs. The main functional entities are as follows.

TThe BAN is mainly used to support heterogeneous paging. In a mobile environment, systems must be energy-efficient since terminals rely on batteries to operate. We expect that wireless IP communicators will be online continuously (i.e., "always on"), although not necessarily communicating most of the time. In essence, mobile hosts will be in an idle state, but passively connected to the network infrastructure. It is then extremely inefficient to have to scan all RANs and wait for a paging message. Moreover, since wireless networks are optimized for special services, they may not be very efficient for paging messages. A wireless network optimized for this kind of traffic is more efficient.

TThe BAN can provide wireless system discovery. The BAN enables common access; every mobile host can use this BAN. The network provides the terminal with information about currently available wireless networks, so the terminal does not have to scan all possible RANs.

IThe BAN is used as a signaling network espe- cially to enable vertical handoffs. Such a dedicated network can provide this service efficiently and securely.

IThe BAN can provide an infrastructure to allow mobile hosts to determine their location. This information can, in turn, be used by the BAN to provide a mobile host with information about available services in its region. Location management becomes further important for roaming and paging.

TThe BAN is used as a medium for most signaling and control messages. This simplifies the design of new wireless access services, since signaling is performed by another entity (the basic access component).

TSince we have a heterogeneous architecture in which multiple RANs can be used (semi) simultaneously, we need to have a network access synchronization mechanism so that a terminal could know when to tune the SDR to another access network. The BAN can provide such a service straightforwardly.

TFinally, the BAN can also be used as a wireless access service when a user uses a BAC standalone. It is, however, primarily suitable for very low-bandwidth messaging services such as short messages.

Because the BAN is mainly used for short messages, speed is of less importance; the total capacity, however, must be sufficient for a large number of mobile hosts.

\section{Functional Entities of Mobile Hosts}

Mobile hosts include all standard transport protocols and wireless-specific control services. Control messages are transparently sent between the core network and mobile hosts' functional entities.

As shown in Fig. 5, a mobile host will contain a BAC with a locator and an SDR-based NI. The BAC is used as a primary component to communicate with the BAN and has an embedded positioning capability offered by the locator (e.g., a GPS receiver). The BAC sends out location update data for paging (coarse update) when the mobile host moves across the paging boundary, and for system discovery (fine update) when the mobile host initializes a call or requires a vertical handoff. The NS is an entity that selects the required access network. It communicates with the RM in the CCN via a BAN to determine which network should be used and when it will be operational.

There will be one or more subsystems for accessing (communicating with) subscribed service systems or RANs. These are indicated as subsystem A, ..., subsystem $N$. When an NI is implemented with SDR technologies, using more than one access network at the same time may be difficult (except for transitional duration required for a vertical handoff). The RM in the CCN distributes the traffic based on user preferences, resources of the common core network including RANs, and local resources of the terminal. The LRM deals with the local resources of the terminal and interacts with the RM in the CCN. Applications should be able to use the infrastructure and specify the traffic and QoS requirements. A $Q o S A P I$ is used by the applications to specify their needs and establish a session. If they do not use this application 
programming interface (API), best-effort mechanisms will be used for their session.

All radio access subsystems (including the BAC) will be equipped with all necessary components to independently operate with the corresponding air interfaces. Inbound (outbound) data will be delivered to (received from) the user equipment central processor (CP). The CP (hosting an embedded operating system with a TCP/IP stack) will coordinate all operations within a mobile host. For example, it will handle the user interface, monitor channels, configure and switch between service subsystems, and so on.

\section{Challenges}

We are currently in the process of designing the architecture, analyzing its effectiveness, implementing basic functions and protocols, and preparing a proof-of-concept demonstration. In the process we have also identified a number of research issues for which no satisfactory solution currently exists.

The envisioned architecture for wireless Internet on heterogonous networks offers real design challenges in many aspects. Its success depends on a careful analysis and design of required protocols and systems. However, we envision prototypes of the architecture implemented with existing technologies, using current research results and protocols. For example, the CCN architecture can be partially based, for example, on Cellular IP or HAWAII. Current two-way paging systems can be referred to implement the BAN. For the external network we rely on existing mechanisms that support mobility such as Mobile IPv6, and existing work on QoS over IP (Diffserv and/or Intserv). As long as proper software radio systems do not exist, we can use multiple radio subsystems instead.

Nevertheless, much challenging work remains to be done on various topics:

ISignaling protocols. The design of the signaling protocols between the mobile host and the $\mathrm{CCN}$ is likely to be based on existing work of protocols enabling micro-mobility. However, the heterogeneous network poses some extra demand on the protocols. For instance, a signaling protocol has to be designed to implement the Network Selection Control that enables the software radio to tune to the right access network at the appropriate times. Since one CCN can support many mobile hosts, scalability and complexity are very important.

TRouting and handoff. Although we may opt for adopting an existing mechanism, there are still various specific problems to be solved (e.g. how to handle differentiated flows). Scalability in the CCN will probably be the major challenge. Hybrid solutions based on existing protocols, like Mobile IPv6 for the top routers in the hierarchy and Cellular IP for the lower routers within the network, will be an option.

IQoS management. For the external network we rely on existing mechanisms. However, how to manage QoS in the core network is still an open question.

TLocation management. To enable the efficient utilization of the heterogeneous network,

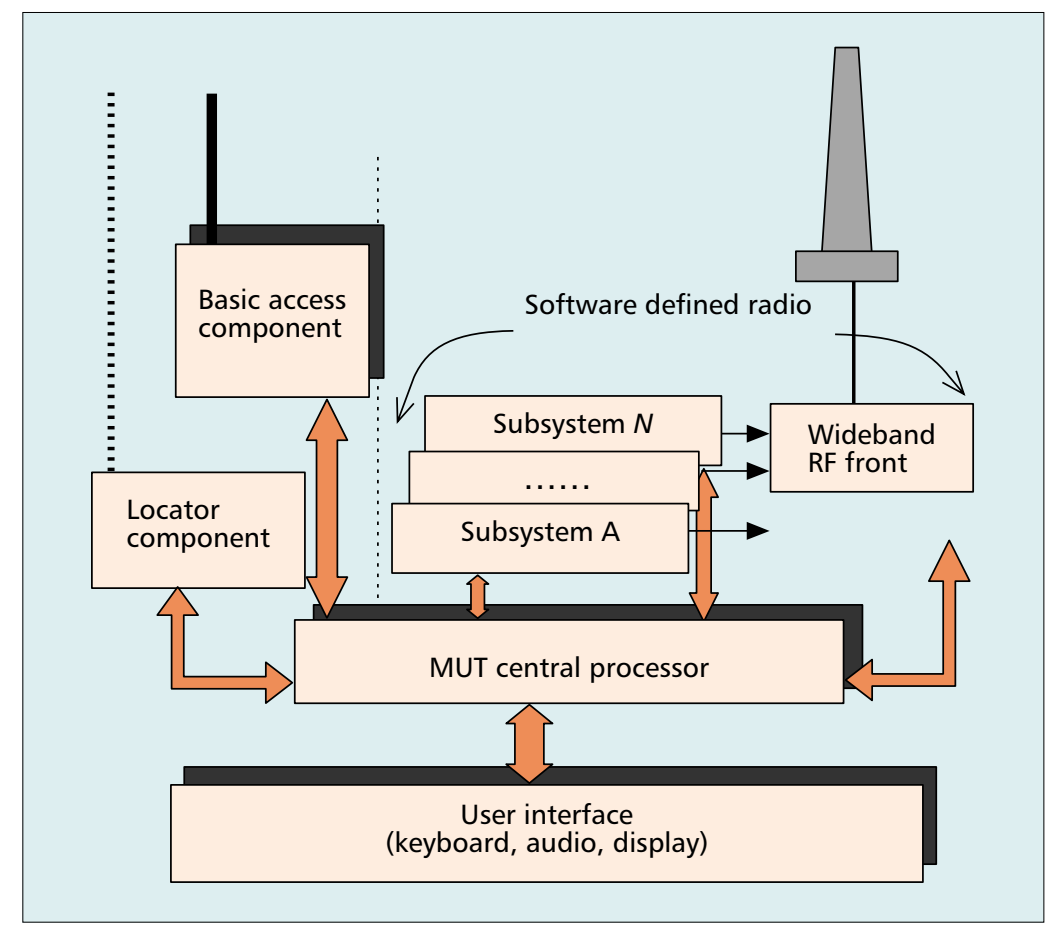

Figure 5. An image of the mobile host.

information about the current location of the mobile hosts is very important. The location mechanism should balance the cost of precision or currency against the cost of location updates. Also, various ways to determine the location of a mobile must be revealed and evaluated.

IMobility manager $(M M)$. This issue is closely related to the previous one. The MM should maintain the database with the location of all mobile hosts in the coverage area of the CCN. The number of entries can be very large. Other tasks of the MM are to support handoffs. Again, this raises the issue of scalability. It is envisioned that the MM is distributed in a hierarchy among various entities each dealing with a smaller region.

IResource manager. This might be the most challenging item since the complexity is large (there are many input parameters), the required performance is high (it should keep up with fast moving mobiles), and the number of sessions on the core network can be very large (a region is envisioned to be as large a metropolitan area). Distributing might be necessary, but is not straightforward. A design with a hierarchy of regional RMs is likely to be appropriate. A proper API and interface, for the user to be in control of what is happening, must be designed.

ISoftware-defined radio. SDR technologies are still in their infancy. A major design challenge here is to minimize the reconfiguration time that is needed.

TSecurity. Mobility exposes mobile host security threats that are not trivially found and solved. Mobile IP already provides several mechanisms that should be adopted. As a first step, all signaling messages should be authenticated and preferably encrypted. This is needed since these signaling messages establish and change network parameters. Encryption is needed to 


As the envisioned
range of the
network should
have the size of a
metropolitan
area, the number
of mobile hosts
and the number
of base stations,
may impose
major
performance
problems for
many of the
above-mentioned
topics.

ensure privacy of, for example, location information. Timeliness of the authentication process is critical due to the requirement of fast handoff control.

Performance and scalability are the most prominent challenges within the CCN. Since the envisioned range of the network should have the size of a metropolitan area, the numbers of mobile hosts and BSs may impose major performance problems for many of the above-mentioned topics.

\section{CONCLUSION}

The presented architecture shows a novel approach to enable the efficient use of available RANs. The basic concept is that each service is delivered via a network that is most efficient to support the service. The result is that the mobile user receives the requested service at the lowest cost, and scarce radio resources are used efficiently. The architecture solves many problems of the wireless Internet over heterogeneous networks.

Currently, a proof-of-concept experimental demonstration system based on the MIRAI architecture is being developed at the Communications Research Laboratory and will be available by the end of the Japanese Fiscal Year 2001 (March 2002). Personal Handyphone System (PHS) and $802.11 \mathrm{~b}$ wireless LAN will be used as two different wireless access technologies in the system. The MUT is currently implemented by integrating two individual PCMCIA modules of PHS and WLAN to meet the schedule and will be replaced by SDR implementation by the end of 2002 . An experimental BAN with the basic functional entities described herein has been designed for operation in the $400 \mathrm{MHz}$ band to support the common signaling of integrated RANs. An adaptive modulation-based physical layer has been designed to support a fixed symbol rate of 19 ksymbols/s in the reverse link and a dynamic time-division-multiple-access-based medium access control protocol has been designed for the implementation. The design of the CCN is based on the concept of region networks. There is a signaling home agent (SHA) designed in each region network to manage location updates, paging, micromobility, and minimum authentication. Modified Cellular IPv6 will be implemented in the region networks. A region registrar has been designed and implemented in the external IPv6 network. A modified Mobile IPv6 protocol will be implemented for handoffs between two CCNs. A related indoor/outdoor test field providing a physical handover environment is also being developed in the Yokosuka Research Park area.

\section{REFERENCES}

[1] R. Keller et al., "Convergence of Cellular and Broadcast Networks from a Multi-radio Perspective," IEEE Pers. Commun., vol. 8, no. 2, Apr. 2001, pp. 51-56.

[2] R. Walsh, L. Xu, and T. Paila, "Hybrid Networks - A Step Beyond 3G," 3rd Int'l. Symp. Wireless Pers. Multimedia Commun.), Bangkok, Thailand, Nov. 2000, pp. 109-14.
[3] C. Perkins, "IP Mobility Support," IETF RFC 2002, Oct. 1996.

[4] A. T. Campbell et al., "Design, Implementation and Evaluation of Cellular IP," IEEE Pers. Commun., vol. 7 no. 4, Aug. 2000, pp. 42-49.

[5] R. Ramjee et al., "IP-based Access network Infrastructure for Next-generation Wireless Data Networks," IEEE Pers. Commun., vol. 7, no. 4, Aug. 2000, pp. 34-41.

[6] R. Braden, D. Clark, and S. Shenker, "Integrated Services in the Internet Architecture: An Overview," IETF RFC 1633, 1994.

[7] S. Blake et al., "An Architecture for Differentiated Services," IETF RFC 2475, 1998.

[8] Daedalus proj., UC Berkeley, http://daedalus.cs.berkeley.edu

[9] Monarch proj., CMU, http://www.monarch.cs.cmu.edu

[10] Magic RANd proj., http://www.tik.ee.ethz.ch/ RANd

[11] V. Rexhepi, G. Karagiannis, and G. Heijenk, "A Framework for QoS \& Mobility in the Internet Next Generation," Proc. EUNICE 2000, Univ. of Twente, Enschede, Netherlands, Sept. 2000.

\section{AdDITIONAL READINGS}

[1] S. Deering and R. Hinden, "Internet Protocol, version 6 (IPv6), Specification," IETF RFC 2460, Dec. 1998.

[2] E. Pitoura, and G. Samaras, "Locating Objects in Mobile Computing," IEEE Trans. Knowledge and Data Eng., vol. 13, no. 4, July, 2001, pp. 571-92.

\section{BIOGRAPHIES}

GANG WU [SM '99] (g-wu@crl.go.jp) received a B.E. degree from Xian Jiaotong University, China, an M.S.E.E degree from Xidian University, China, and a Ph.D. degree from Shizuoka University, Japan, in 1984, 1987, and 1994, respectively. From 1987 to 1989 he was a faculty member of information engineering at Xidian University. He joined the Communications Research Laboratory, Japan, in 1994, where he is a senior researcher. He was a visiting researcher at WINLAB, Rutgers University, New Jersey, during academic year 1998. Since 1994 he has been involved in R\&D projects related to broadband mobile communication systems in microwave band, ultra-high-speed wireless LANs in millimeter-wave band, infostation systems, broadband wireless access systems using stratospheric platforms, and seamless integration of wireless access networks. He has authored more than 60 reviewed publications in above areas. He received the PAACS Friendship Award from the IEICE in 1994 and is serving as Secretary of the Radio Communication Systems Committee of IEICE Communications Society.

PAUL J.M. HAVINGA (havinga@cs.utwente.nl) received his B.S. degree in computer engineering in 1985. Since 1985 he has been working at the Computer Science Department of the University of Twente, the Netherlands. His research has been on parallel processing, ATM switching, and mobile computing and wireless communication. He has been a visiting researcher at the University of Pisa in 1998, and the Communications Research Laboratory, Japan, in 2000. He received his Ph.D. on mobile multimedia systems in 2000, and was awarded with the DOW Dissertation Energy Award for this work. His research interests are in the area of energy-efficient architectures and protocols, wireless communication networks, personal communication systems, wireless sensor networks, multimedia communication, and reconfigurable systems.

MitsuHIKo MızUno [M '82] (mizuno@crl.go.jp) received his B.Eng., M. Eng., and D.Eng. degrees in electronics from Kyoto University in 1976, 1978 and 1989 respectively. In 1978 he joined Communications Research Laboratory (CRL), Ministry of Posts and Telecommunications, where he has been working on mobile communications. In 1988 he was a guest researcher at Swedish Telecom Radio. From 1995 to 1999 he was head of the Radio Transmission Department of YRP Mobile Telecommunications Key Technical Labs. He is currently group leader of the Wireless Networks Integration Group, CRL, and Independent Administrative Institution. He also holds the post of visiting professor at Yokohama National University. 